\title{
Dosage de l'humidité résiduelle des vaccins lyophilisés par le réactif de Karl Fischer Technique et causes d'erreurs
}

\author{
par P. CAISEY $\left({ }^{*}\right)$ et J. BALIS $\left({ }^{*}\right)$
}

\begin{abstract}
RESUME
Les auteurs décrivent une technique rapide de dosage de l'humidité résiduelle des vaccins lyophilisés par le réactif de Karl Fischer.
\end{abstract}

L'humidité résiduelle dans un produit lyophilisé revêt une très grande importance car son taux, en général très faible, est un des éléments majeurs intervenant dans la conservation des vaccins bactériens et viraux. La connaissance précise de ce facteur est indispensable si l'on veut comparer des échantillons de différents lots en fonction de paramètres tels que la température ou le temps.

Le réactif de Karl Fischer, par sa sensibilité, permet un dosage très précis de cette humidité résiduelle.

Malheureusement, sa mise en application est souvent entachée de multiples erreurs dues à la présence de faibles quantités d'eau dans l'atmosphère ou dans les récipients utilisés pour le titrage des réactifs. La technique que nous avons utilisée élimine pratiquement ces sources d'erreurs et nous a permis d'obtenir de bons résultats à l'aide d'un matériel de laboratoire courant.

\section{PRINCIPE DE LA METHODE}

L'anhydride sulfureux est oxydé par l'iode en présence d'eau. Il y a formation d'acide

(*) Veterinary Institute, P.O. Box 19, Debré-Zeit. Ethiopie. sulfurique et d'acide iodhydrique. Cette réaction étant réversible dès que la teneur en acide dépasse 0,05 p. 100 , on la rend complète par addition de pyridine et de méthanol qui neutralisent les acides formés. On aboutit ainsi à l'équation suivante:

$$
\begin{gathered}
\mathrm{H}_{2} \mathrm{O}+\mathrm{I}_{2}+\mathrm{SO}_{2}+3 \mathrm{C}_{5} \mathrm{H}_{5} \mathrm{~N}+\mathrm{HCH}_{2} \mathrm{OH}= \\
2 \mathrm{C}_{5} \mathrm{H}_{5} \mathrm{NHI}+\mathrm{C}_{5} \mathrm{H}_{5} \mathrm{NSO}_{4} \mathrm{HCH}_{3}
\end{gathered}
$$

Une molécule d'iode correspond à une molécule d'eau, il est donc possible de calculer la teneur en eau d'un échantillon par la mesure de la quantité d'iode réduit. Quand toute l'eau est combinée, le point final de la réaction est indiqué par la première goutte de réactif en excès.

Il peut être noté colorimétriquement :

- soit par voie directe : virage du jaune citron au brun clair, en présence d'un témoin de coloration. C'est la méthode que nous allons décrire;

- soit par voie indirecte, par un dosage en retour : le virage est alors plus net mais le point neutre est en fait dépassé.

\section{REACTIFS}

\section{Alcool méthylique}

Il devrait être pratiquement anhydre. Ceci 
étant très difficile à obtenir, on titre la quantité d'eau qu'il contient et on effectue la correction au cours des dosages.

\section{Réactif de Karl FISCHER}

On trouve dans le commerce une «solution pour réactif de Karl FISCHER » (*) dont la composition est la suivante :

Pyridine anhydre : $270 \mathrm{ml}$

Méthanol anhydre: $670 \mathrm{ml}$

Anhydride sulfureux : $64 \mathrm{~g}$

Le réactif de Karl FISCHER est obtenu en ajoutant $85 \mathrm{~g}$ d'iode à un litre de cette solution. Le mélange, une fois effectué, est très sensible à l'humidité. Il doit être conservé dans des flacons parfaitement bouchés et à l'abri de la lumière. Il doit de plus être titré avant chaque utilisation.

Théoriquement la quantité d'iode nécessaire et suffisante pour oxyder $64 \mathrm{~g}$ d'anhydride sulfureux serait de $254 \mathrm{~g}$, mais alors la réaction serait moins rapide : en effet l'excès d'anhydride sulfureux d'une part et de pyridine d'autre part sont destinés à déplacer l'équilibre dans le sens de la consommation d'eau.

Dans certains cas, pour obtenir une plus grande sensibilité, il est possible d'opérer avec un réactif de Karl FISCHER de titre plus faible, c'est-à-dire contenant moins de $85 \mathrm{~g}$ d'iode par litre.

\section{MATERIEL}

Il s'agit d'un matériel de laboratoire courant :

- Une balance de précision type Mettler;

- Un montage classique contenant du chlorure de calcium destiné à dessécher l'air que l'on introduit dans les flacons;

- Deux rhéomètres étalonnés pour les opérations de dilution et de transvasement de l'alcool méthylique;

- Des seringues en plastique, type tuberculine, graduées au centième, pour mesurer les volumes de réactif de Karl FISCHER que I'on a utilisé;

- Des flacons de 20 et $50 \mathrm{ml}$ parfaitement secs, obturés par des bouchons de type pénicilline et capsulés; la meilleure techni-

(*) Solution pour réactif de Karl FISCHER suivant norme NFT 20-052; PROLABO, 12 rue Pelée, PARIS. que consiste à inclure dans le lot de vaccin à lyophiliser une vingtaine de flacons vides et à les traiter dans les mêmes conditions que le lot de vaccin. Ces flacons sont utilisés pour le titrage des réactifs et pour les dilutions;

- Deux flacons contenant l'un le réactif de Karl FISCHER, l'autre l'alcool méthylique, bouchés hermétiquement et munis d'un joint de caoutchouc perforable. Le prélèvement doit être effectué sans ouvrir le flacon, le vide créé étant comblé par de l'air passé sur colonne desséchante.

\section{TITRAGE DES REACTIFS}

Les titrages de l'alcool méthylique et du réactif de Karl FISCHER sont effectués conjointement :

\section{Ier temps}

A l'aide d'un rhéomètre, introduire un volume $a \mathrm{ml}$ d'alcool méthylique dans un flacon de $20 \mathrm{ml}$; puis avec une seringue type tuberculine graduée au centième, ajouter, progressivement et en agitant, le réactif de Karl FISCHER jusqu'à l'apparition d'une coloration brun clair. Cette coloration doit persister au moins $5 \mathrm{mi}-$ nutes. Soit $x \mathrm{ml}$ le volume de réactif ajouté.

\section{$2^{\text {e }}$ temps}

Dans des flacons de $50 \mathrm{ml}$ et à l'aide d'un second rhéomètre, réaliser une dilution d'eau distillée à 1 pour 1000 à partir du même alcool méthylique. Puis répéter le titrage précédent sur un volume $a \mathrm{ml}$ de cette dilution, $y$ étant le volume de réactif ajouté, on en déduit que $1 \mathrm{ml}$ de réactif de Karl FISCHER correspond à $\frac{a}{y-x} \mathrm{mg}$ d'eau.

\section{DOSAGE DE L'HUMIDITE RESIDUELLE DANS UN VACCIN LYOPHILISE ET CONSERVE EN FLACON SOUS VIDE}

On opère de la façon suivante :

$1^{\circ}$ « casser » le vide avec de l'air passé sur colonne desséchante;

$2^{\circ}$ Peser le flacon de vaccin (avec son contenu, son bouchon, sa capsule). Soit $P$ le poids obtenu;

$3^{\circ}$ Introduire l'alcool méthylique titré à l'aide 
d'un rhéomètre $(4 \mathrm{ml}$ pour un flacon de $20 \mathrm{ml}$ ou $1 \mathrm{ml}$ pour un flacon de $5 \mathrm{ml}$ ); tille;

$4^{\circ}$ Agiter jusqu'à désagrégation de la pas-

$5^{\circ}$ Avec une seringue en plastique, type tuberculine, graduée au centième, ajouter, progressivement et en agitant, le réactif de Karl FISCHER jusqu'à virage au brun clair. Cette coloration doit persister au moins 5 minutes. Soit V le volume de réactif utilisé;

$6^{\circ}$ Ouvrir le flacon, le nettoyer et le rincer à l'eau distillée ainsi que le bouchon et la capsule. Sécher l'ensemble à l'étuve;

$7^{\circ}$ Peser le flacon vide avec son bouchon et sa capsule. Soit $p$ le poids obtenu.

$8^{\circ}$ Calcul de la teneur en eau : le réactif de Karl FISCHER ajouté s'est combiné d'une part à l'eau de la pastille, d'autre part à l'eau de l'alcool méthylique introduit dans le flacon. Connaissant la teneur en eau de cet alcool, on en déduit $v$, volume de réactif de Karl FISCHER qui s'est combiné à l'eau apportée par l'alcool méthylique. Sachant que $1 \mathrm{ml}$ de réactif de Karl FISCHER correspond à $\frac{a}{y-x} \mathrm{mg}$ d'eau, on en déduit le pourcentage d'eau contenue dans l'échantillon:

p. 100 eau $=\frac{\mathrm{V}-v}{\mathrm{P}-p} \cdot \frac{a}{y-x} \cdot 100$

Les volumes sont exprimés en millilitres et les poids en milligrammes.

\section{CAUSES D'ERREURS}

\section{A. Erreurs systématiques}

Elles doivent être évitées et nous allons les énumérer avec pour chacune un exemple mettant en valeur leur importance.

$1^{\circ}$ Ne pas omettre de «casser $»$ le vide avec de l'air desséché. En effet, d'une part $1 \mathrm{ml}$ d'air pèse environ $1,3 \mathrm{mg}$, soit une erreur de pesée de $26 \mathrm{mg}$ pour un conditionnement courant de $20 \mathrm{ml}$; d'autre part, à demi saturation et à la température du laboratoire, $20 \mathrm{ml}$ d'air contiennent déjà $0,22 \mathrm{mg}$ d'eau. Ce chiffre est capable de fausser les mesures puisque la quantitê d'eau résiduelle dans une pastille de $160 \mathrm{mg}$ est d'environ $1,7 \mathrm{mg}$.

$2^{\circ}$ Il est indispensable de peser la pastille lyophilisée à l'intérieur du flacon car :
- Il reste toujours des fragments adhérents aux parois. Une vérification sur 49 échantillons nous a donné une différence de poids de 14 p. 100 environ;

- Une pastille extraite du flacon reprend très rapidement une humidité importante. Sur trois lots de 10 échantillons chacun, nous avons obtenu les différences suivantes:

$1^{\text {er }}$ lot : vide "cassé 》 sur air desséché : humidité 1,18 p. 100 ;

$2^{\mathrm{c}}$ lot: ouverture du flacon, sortie et pesée de la pastille, le tout en 3 minutes : humidité 1,80 p. 100 ;

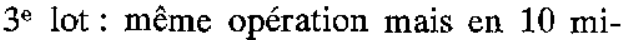
nutes : humidité : 3,10 p. 100.

$3^{\circ}$ Les flacons utilisés pour le titrage des réactifs doivent être parfaitement secs. Il est possible de le vérifier par addition de quelques gouttes de réactif de Karl FISCHER.

\section{B. Erreurs expérimentales}

Reprenons la formule: Humidité résiduelle en p. $100=\frac{\mathrm{V}-v}{\mathrm{P}-p} \cdot \frac{a}{y-x} \cdot 100$

L'erreur relative est égale à la somme des erreurs relatives de chacun des éléments. Mais, d'une part dans cette formule le facteur $\frac{a}{y-x}$ peut être déterminé avec précision en faisant la moyenne d'une série de titrages. Ce facteur sera le même pour un ensemble de mesures d'humidité. D'autre part l'erreur commise sur $\mathbf{P}-p$ est très faible, la balance de Mettler étant sensible au dixième de milligramme. En définitive, il ne reste comme source principale d'incertitude relative que :

$$
\frac{d \mathrm{~V}}{\mathrm{~V}}+\frac{d v}{v} ; d \mathrm{~V} \text { et }
$$

$d v$ étant de l'ordre de $0,02 \mathrm{ml}$ il est possible d'obtenir une valeur de l'humidité résiduelle avec une précision de 0,06 p. 100 lorsqu'il sagit d'une pastille de $100 \mathrm{mg}$, cette précision atteignant 0,02 p. 100 pour un échantillon de $700 \mathrm{mg}$.

\section{CONCLUSION}

Le réactif de Karl FISCHER permet un dosage rapide et précis de l'humidité résiduelle dans un vaccin lyophilisé. Cependant, en raison 
de sa très grande affinité pour l'eau, il est nécessaire d'effectuer toutes les manipulations en atmosphère desséchée. Le mode opératoire que nous avons suivi permet d'obtenir des résultats satisfaisants à l'aide d'un matériel de laboratoire courant.

\section{SUMMARY}

Residual moisture evaluation in lyophilized vaccines by Karl Fischer's method. Technique and causes of mistakes

The authors describe a rapid technique of residual moisture evaluation in lyophilized vaccines by Karl Fischer's method.

\section{RESUMEN}

Dosaje de la humedad residual de las vacunas liofilisadas por el reactivo de Karl Fischer. Técnica y causas de errores

Los autores describen una técnica rápida de dosaje de la humedad residual de las vacunas liofilisadas por el reactivo de Karl Fischer. 